\title{
Les Cahiers d'exercices \\ Prolifération d'une nouvelle (?) forme de manuels scolaires. L'exemple du Québec ${ }^{1}$
}

\section{Paul Aubin}

\section{RÉSUMÉ}

Les premiers cahiers d'exercices apparaissent au Québec en 1882. Peu nombreux au début, ils occupent une place de plus en plus grande après la Seconde Guerre mondiale, à un point tel que, durant les deux dernières décennies, ils rivalisent avec le manuel traditionnel, tout au moins dans certaines disciplines. On en compte maintenant plus de 2500.

Publiés, il va de soi, à l'intention du monde scolaire, les cahiers d'exercices en subissent les mêmes contraintes, à commencer par la politique d'approbation.

Durant la première période (1880-1960), ils connaissent une augmentation lente mais constante; cependant le manuel classique domine encore le marché.

La deuxième période (1960-2000) voit les cahiers d'exercices gruger de plus en plus le marché traditionnel du manuel scolaire. De nouvelles théories pédagogiques et de nouveaux programmes favorisent cette explosion; ainsi, durant la décennie 1990, seulement $45 \%$ des imprimés pédagogiques sont des manuels scolaires. Ce changement spectaculaire en nombre par rapport au manuel classique s'accompagne d'un changement tout aussi spectaculaire dans la forme et dans le contenu.

\section{ABSTRACT}

The first school exercise books appeared in Québec in 1882. Initially small, their production took off after World War II to such an extent that it has rivalled that of traditional textbooks over the past twenty years, at least in some disciplines. There are now more than 2,500 different exercise books.

Published by definition for use in the schooling world, exercise books are subjected to the constraints imposed, notably through the state approval process for teaching materials in print.

The first period (1880-1960) witnessed a slow but steady increase in the publication of exercise books; however, traditional textbooks still dominated in both the classroom and the market place.

The second period (1960-2000) saw the exercise book encroach more and more upon the dominance of the textbook. New currents in education and new programmes created conditions 
favourable to an explosion in the use of exercise books; thus, during the 1990s, only 45 percent of schoolbooks were exercise books. Certainly, the change in numbers was spectacular, but no less spectacular was the transformation of form and content.

\section{Introduction}

De tout temps, les élèves ont " orné » leurs manuels de graffitis, dessins, commentaires, passages soulignés, et cela, en toute impunité, d'autant plus qu'ils en étaient les propriétaires. La nouveauté consistera à produire des imprimés destinés à l'enseignement, mais dans lesquels on réserve explicitement des espaces permettant à l'élève d'écrire ses réponses : dorénavant, l'élève est appelé à écrire dans ses manuels! Ces « cahiers d'exercices " pour employer la formule consacrée deviennent donc des " cahiers de devoirs " remplaçant, jusqu'à un certain point, les «cahiers brouillon » d'autrefois et offrant des possibilités nouvelles : non seulement y inscrit-on ses réponses, mais on peut aussi s'y exprimer par des dessins et, parfois, les mutiler pour la bonne cause lorsqu'on demande à l'élève de découper certaines pages suivant le pointillé pour produire des formes géométriques. D’une timide apparition durant la décennie 1880, ils en arrivent à devenir, depuis quelques années, un des outils pédagogiques les plus utilisés dans l'enseignement, témoignant à la fois de nouvelles approches pédagogiques et de la volonté (ou nécessité?) des autorités gouvernementales de composer avec un produit qui a visiblement trouvé la cote tant auprès des professeurs que des élèves.

Le présent article tentera d'expliquer le contexte dans lequel s'est développé le cahier d'exercice ainsi compris et d'illustrer quelques-unes des formes et des applications pédagogiques qu'il a explorées. Si nous avons produit des centaines d'études sur l'histoire du manuel scolaire québécois, nous n'avons pratiquement jamais abordé l'histoire du cahier d'exercices. Or cette approche chronologique s'impose d'autant plus que ce type d'outil pédagogique, de marginal qu'il était au début, est en passe de se tailler une place majeure dans la panoplie des instruments de transmission du savoir en classe. Après un bref rappel des principales étapes de l'histoire du manuel scolaire tel qu'entendu au sens large du terme, j'aborderai le cas du cahier d'exercices en précisant les grandes phases de son développement, les facteurs qui sont à l'origine de son développement et les changements qu'il a connus tant dans la présentation matérielle que dans les travaux qu'il exige des élèves.

Le manuel scolaire naît au Québec en même temps que l'imprimerie : le premier livre qu'on y publie paraît en 1765 et c'est un catéchisme. ${ }^{2}$ D'une production forcément restreinte dans les premiers temps - 18 titres publiés au $18^{\mathrm{e}}$ siècle — nous passons à un corpus de plus de 2000 titres durant la décennie $1990 .^{3}$ Cette augmentation fulgurante ne s'explique pas que par la croissance démographique : il faut aussi prendre en compte la multiplicité des disciplines nouvelles qui se sont ajoutées en réponse au développement des besoins de la société. Cette production s'adresse d'abord à la majorité francophone sans négliger pour autant la principale minorité, les anglophones, tout en essayant de tenir compte des autres minorités établies depuis longtemps - Amérindiens, Esquimaux, Juifs — et également des nouvelles minorités qui depuis quelque temps colorent notre paysage. 
Les premières structures stables en matière d'éducation apparaissent en 1841 avec la création du Bureau d'Éducation mais surtout en 1856 avec la mise sur pied du Conseil de l'Instruction publique qui reçoit, entre autres mandats, celui d'établir des programmes et de voir à ce que les manuels qui en découlent respectent à la fois les contenus pédagogiques et les croyances religieuses diverses : c'est la politique d'approbation toujours en vigueur. Essentiellement affaire d'Églises - catholique, protestante — particulièrement à partir de 1875 alors qu'est aboli l'éphémère ministère de l'Éducation institué en 1867, l'administration scolaire s'est laïcisée, notamment avec la création de l'actuel ministère de l'Éducation en 1964 qui a repris à son compte les politiques de son prédécesseur, le Conseil de l'Instruction publique, notamment le contrôle sur les imprimés circulant dans les écoles.

Deux de ces politiques - pour ne pas dire batailles — auront des incidences directes sur les manuels et particulièrement sur les cahiers d'exercices. On a évoqué plus haut le système d'approbation instauré graduellement à partir de 1840 : tout manuel, pour avoir droit de circuler dans les écoles, doit être " approuvé " par l'autorité gouvernementale ; le défaut d'approbation signifie, tout au moins théoriquement, la fermeture du marché. En effet, le Ministre dispose d'une arme redoutable pour appliquer la politique d'approbation : la gratuité des manuels instaurée graduellement à partir de 1944. Les commissions scolaires fournissent aux élèves les manuels et se font rembourser par le ministère, sur présentation des factures, les sommes engagées, remboursement qui ne porte que sur les manuels approuvés. ${ }^{4}$

Un nouveau type de manuels, les cahiers d'exercices, imprimés destinés à l'enseignement mais dans lesquels on réserve des espaces permettant à l'élève d'écrire ses réponses, apparaît en 1882 — une dizaine sont publiés durant cette décennie — alors que durant la décennie 1990 on en recense près de 1000 (fig. 1).

Destinés à l'ensemble des élèves, ils contiennent une nette prédominance des textes en français — $94 \%$ - sans oublier pour autant les minorités (fig. 3) ${ }^{5}$ : des cahiers de calligraphie pour l'hébreu sont publiés en $1917^{6}$ et les Inuit ont droit à un même traitement en 1966.7 De même, on comprend que les disciplines "lourdes " accaparent la majorité des publications : $40 \%$ pour l'enseignement du français, $22 \%$ pour l'arithmétique et $8 \%$ pour les sciences humaines (fig. 4).

\section{0-1960 — Les Balbutiements}

De 1880 (fig. 1) à la fin de la décennie 1950, la production de cahiers connaît une progression, lente d'abord et plus accentuée à la fin de la période avec 79 titres durant la décennie 1950. Au fil des ans, non seulement produit-on plus de cahiers, mais on a tendance à leur confier un rôle de plus en plus grand par rapport au manuel traditionnel : alors que durant la décennie 1880, les cahiers représentent $6 \%$ des manuels, ce ratio monte à $10 \%$ durant la décennie $1950^{8}$ (fig. 2). D’où des questions d'ordre quantitatif, mais surtout d'ordre qualitatif : les cahiers favorisent-ils la réflexion ou se contentent-ils de tabler sur un effort intellectuel réduit au minimum?

Il nous faut bien constater que, dans certains cas, on ne favorise guère la créativité de l'élève, comme dans cet album d'histoire sainte où le travail se résume à colorier le 


\section{Cahiers}

Nombre par décennie

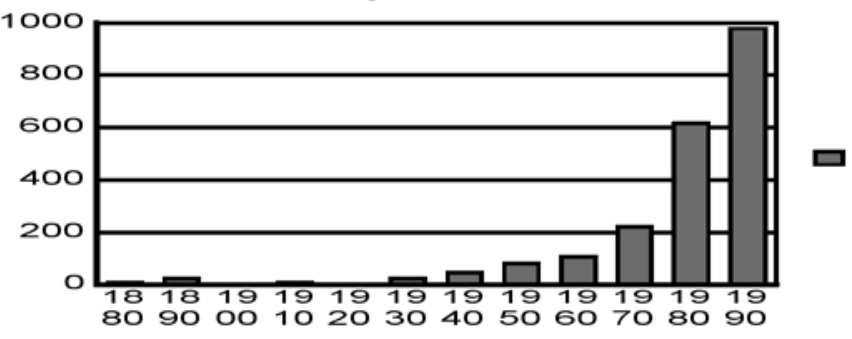

Fig. 1

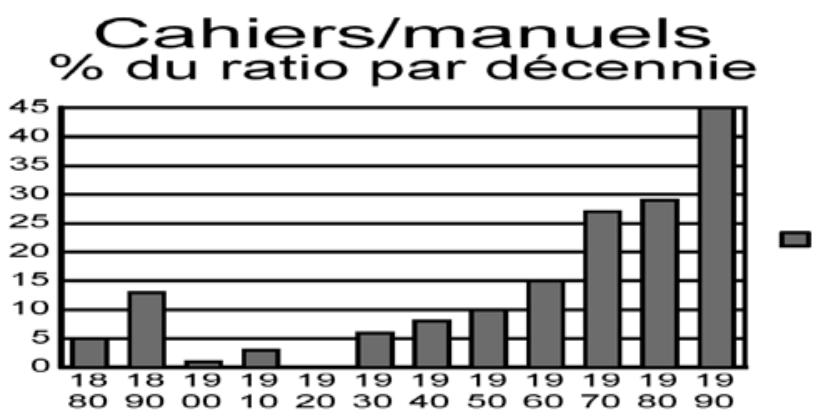

Fig. 2

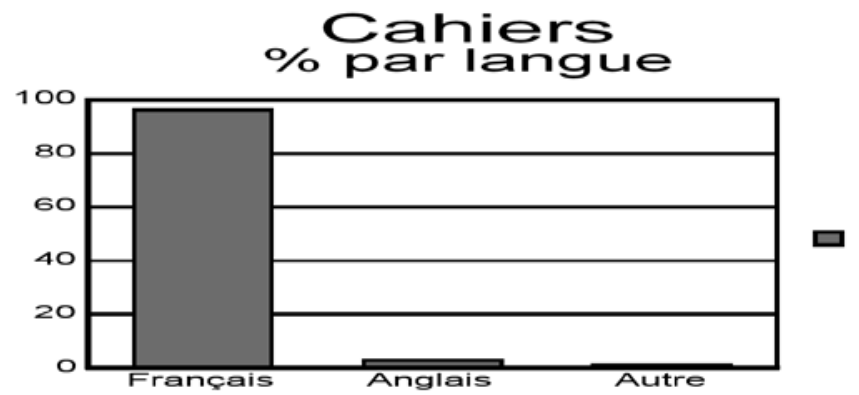

Fig. 3

\section{Cahiers \\ \% par discipline}

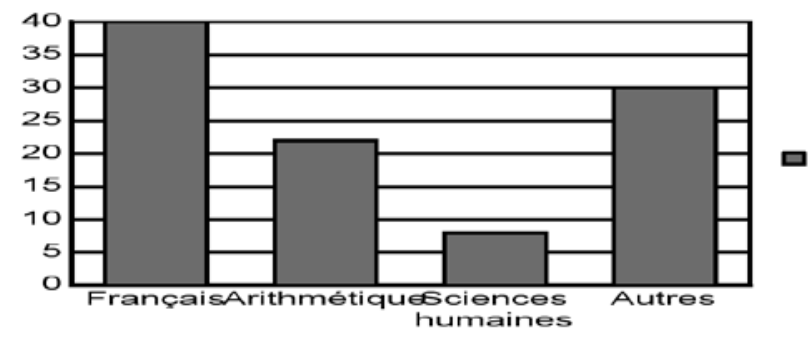

Fig. 4 
dessin, et encore : on va jusqu’à indiquer les couleurs appropriées! ${ }^{9}$ Émigré de France, professeur de dessin, auteur de manuels à la fin du XIX ${ }^{\mathrm{e}}$ siècle, "inspecteur général de l'enseignement du dessin pour la province de Québec ", Edmond-Marie Templé produit des feuilles volantes présentant, à gauche, un dessin qu'il faut reproduire exactement à droite : l'enfant n'est qu'un exécutant. ${ }^{10}$ Dans tel cahier en histoire du Canada, on pose à l'enfant des questions précises, ne commandant aucun développement et dont la réponse se trouve explicitement dans son manuel; ailleurs, on pousse la " sollicitude " plus loin en indiquant à quelle page du manuel trouver la réponse! ${ }^{11}$ Faut-il y voir une démission des auteurs devant la paresse innée des enfants? Le supérieur d'une communauté religieuse qui produit elle-même ses propres manuels estime à la toute fin de la décennie 1870 que les élèves livrent " un travail matériel bien satisfaisant, mais rien ou presque rien pour l'intelligence. ${ }^{12}$ Ce type de cahiers justifie le combat que des pédagogues d'avant-garde menaient et, il faut bien l'admettre, avec des succès plutôt relatifs; qu'on pense à la dénonciation, en 1869 d'un enseignement privilégiant la mémorisation et qu'on qualifiait de " vice dans nos écoles. " ${ }^{13}$ Soixante ans plus tard, le plus éminent scientifique au Québec francophone, le frère MarieVictorin, avertissait les pédagogues : « [...] si l'on n'y prend garde, le livre, le manuel scolaire en particulier, cesse vite d'être un miroir pour devenir un écran, et qu'au lieu d'élargir la pensée, il peut facilement la comprimer, la restreindre, la cadenasser dans la terrible prison des mots. " ${ }^{14}$

Tout n'est quand même pas si sombre. Notons d'abord que certaines disciplines n'exigent que des opérations mécaniques : pensons aux cahiers de calligraphie comportant un en-tête avec quelques mots suivis d'un certain nombre de lignes sur lesquelles on doit reproduire le modèle le plus fidèlement possible. ${ }^{15}$ Cas unique que celui de la comptabilité dont l'apprenti s'attend à remplir, plus tard, des cahiers de colonnes de chiffres; rien de plus normal que de lui fournir, comme cahier d'exercices, des modèles identiques à ceux avec lesquels il gagnera sa vie. ${ }^{16}$ Les tests objectifs — vrai ou faux — ou l'appariement de deux membres de phrases exigent un minimum de réflexion et peuvent être employés dans toutes les disciplines, y compris dans l'enseignement de la religion; ${ }^{17}$ de même, les questions à choix multiples font appel à un minimum de réflexion. ${ }^{18}$ Le calcul de base-les quatre opérations fondamentales - suscite un certain nombre de cahiers, et ce, dès l'apparition de ce type d'outils pédagogiques en $1882 .{ }^{19}$

Enfin, et c'est consolant, cette première période voit aussi l'apparition d'un bon nombre de cahiers d'exercices faisant appel aux facultés d'analyse de l'élève. L'enseignement des langues nous en offre un premier exemple, notamment en anglais durant la décennie 1930, quand on demande aux élèves de remplir les vides dans un texte, soit la formule "fill the blanks. ${ }^{20}$ L'enseignement du français, à la même époque, utilise une formule similaire; ${ }^{21}$ dans les deux cas on traduit la vision normative de l'enseignement des langues qui prévalait à l'époque. Au tournant de la décennie 1950, on voit émerger une nouvelle approche : en plus de devoir remplir les blancs, l'élève doit rédiger de courtes phrases en anglais. ${ }^{22}$

D'autres formules font leur apparition ou tout au moins sont utilisées de façon plus pédagogique, particulièrement le dessin en enseignement de la religion. Soit 
qu'on demande d'analyser un dessin soit que l'élève soit appelé à s'exprimer, à son choix, de deux façons différentes : rédiger un texte ou faire un dessin; on peut aussi céder à la facilité du moralisme : "Tout en coloriant [le dessin représente un enfant combattant les tentations représentées par des serpents], pense à une belle victoire que tu pourrais remporter [...]. ${ }^{23} \mathrm{C}^{\prime}$ est surtout en géographie que les auteurs font preuve d'imagination pour solliciter le travail personnel. Dès 1882 on demande à l'élève de s'inspirer de modèles de géographie locale pour dessiner le plan de sa classe, de son école et de sa paroisse; les mêmes pédagogues lui demandent aussi de dessiner de mémoire des cartes. ${ }^{24}$ Plus tard, on présente une photographie aérienne d'une région que l'on fait suivre du dessin qui la traduit; on demande ensuite à l'élève de refaire le même dessin, l'initiant ainsi à la géographie physique. ${ }^{25}$ On pourra même l'initier à l'interprétation de la stratigraphie. ${ }^{26}$ Un auteur réserve des pages pour coller des photos de paysage prises dans les journaux, transformant le cahier d'exercices en "scrap-book", ce qui n'est pas son utilisation optimale. ${ }^{27}$ Enfin, durant la décennie 1950, les cahiers rendent compte d'un mouvement qui ira s'amplifiant par la suite, soit le décloisonnement entre les disciplines : en histoire du Canada on fait appel aux connaissances en géographie pour situer les théâtres d'opération d'une guerre alors qu'en géographie l'enfant devra avoir recours à ses connaissances en histoire pour comprendre l'évolution d'un territoire donné. ${ }^{28}$ Notons, durant la même décennie, l'arrivée d'un nouveau type de cahier qui connaîtra, lui aussi, une grande vogue, et qui est un exemple de discipline où le cahier d'exercices est essentiel : l'enseignement des sciences - ici la chimie - où le cahier sert à transcrire les résultats des expériences. ${ }^{29}$

Autre signe d'un renouveau pédagogique, les cahiers commencent, au tournant de 1950, à offrir des jeux éducatifs qui peuvent à l'occasion impliquer une équipe d'élèves : l'école ne doit pas être nécessairement triste! En 1948, on insère dans un cahier dédié à l'enseignement du calcul des cartons plus rigides destinés à être découpés suivant un pointillé : d'un côté on indique une opération de soustraction et de l'autre côté on écrit la réponse. ${ }^{30}$ Encore pour l'enseignement du calcul, on apprend à l'enfant à " lire » les chiffres en lui faisant réaliser un dessin. ${ }^{31}$ En français, on utilise une variante des mots croisés. ${ }^{32}$

Les cahiers rendent compte non seulement des pratiques pédagogiques, mais aussi des idéologies que les manuels véhiculent. Longtemps étudiée - et enseignée — dans l'optique de la religion, l'histoire du Canada offre des cahiers qui en témoignent comme celui dans lequel, après le nom de chacun des "saints Martyrs Canadiens" l'élève est appelé à " écrire une petite prière à chacun. " ${ }^{33}$

La période qui s'achève avec la fin de la décennie 1950 a donc vu l'apparition et un début de développement des cahiers d'exercices; encore relativement peu nombreux, ils ne suscitent que peu de commentaires sur leur usage. Parfois un conseil pratique à l'intention des professeurs : sur la dernière page de chaque " key " ( livre du maître ») d'une série destinée à l'apprentissage de l'anglais, on lit : " Les feuilles corrigées devraient être détruites. " ${ }^{34}$ L'auteur d'une Méthode pour apprendre rapidement l'anglais vante, en 1945, l'utilisation de ses cahiers auprès des professeurs, sa clientèle cible : "Avantages - Cette nouvelle méthode d'anglais : 1 - épargne du temps aux professeurs et aux élèves; 2 - est parfaitement graduée; 3 - intéresse grandement les élèves et 
ainsi facilite la discipline; 4 - présente aux maîtres et aux maîtresses une préparation de classe pratique et efficace; 5 - repose le professeur sans que cela soit au détriment de l'écolier. $"{ }^{35}$ En 1952, dans un "guide du maître ", on rappelle au professeur que "Ces notes ne sont que pour servir dans l'appréciation du travail personnel [souligné dans le texte] de l'élève. Toute réponse intelligente et juste devra être acceptée. On laissera les enfants s'exprimer spontanément, dans leurs mots et dans le style rapide des tests, surtout aux endroits où peu d'espace est alloué. " ${ }^{36}$ Et la mémorisation, toujours valorisée, trouve son compte dans l'utilisation des cahiers, particulièrement en histoire : "Remarque. Les devoirs d'histoire de ce fascicule devront être rédigés soigneusement et corrigés scrupuleusement. À la fin de l'année, le fascicule terminé constitue un recueil précieux des parties les plus importantes du programme et peut donc être utilisé pour une récapitulation. $»^{37}$

Suscitant peu de commentaires chez les pédagogues, les cahiers d'exercices attirent également peu d'attention de la part des autorités gouvernementales. On en trouve une seule mention dans la littérature : en 1953 la "Commission des manuels [...] recommande qu'il soit strictement défendu d'utiliser dans les écoles, comme livres de l'élève, les résumés de cours, les cahiers d'exercices, les questionnaires d'examen ou tout autre matériel de classe non autorisé par le Comité catholique [...] avec, comme exemple, 50 devoirs sur les verbes "; on peut douter de l'efficacité de la directive car ce même cahier est réimprimé en $1957 .{ }^{38}$

N'occupant toujours qu'une place secondaire dans la panoplie des instruments à la disposition des professeurs, les cahiers d'exercices n'en ont pas moins expérimenté différentes formes; la période qui suit en verra l'âge d'or tant par leur importance numérique que par la diversité de leur présentation.

\section{0-2000 — L'âge d'or}

Avec l'arrivée de la décennie 1960, le cahier d'exercices prend son véritable envol, tant en nombre absolu qu'en importance relative par rapport au manuel traditionnel : pour la première fois il franchit le cap de 100 (fig. 1) et il passe de $15 \%$ d'occupation du champ des écrits pédagogiques à près de $45 \%$ pour la décennie 1990 (fig. 2). Signe des temps : l'immigration massive nécessite l'adaptation, pour les nouveaux arrivants, de formules éprouvées; appelés à analyser le même dessin, les francophones de souche se font interroger sur "vilebrequin et mèche ", termes qui n'apparaissent pas dans la version destinée à ceux qui en sont encore à s'initier aux rudiments du français. ${ }^{39}$ L'alphabétisation des adultes recourt également aux cahiers : la différence d'âge de la clientèle n'a rien à voir avec cet instrument pédagogique. ${ }^{40}$ Non seulement les cahiers s'adressent-ils à de nouvelles clientèles mais ils occupent un espace traditionnellement hors-école : les vacances. Après un généraliste Mon cahier de vacances-3e année vers 1965, on passe à des cahiers spécialisés, comme en lecture où l'enfant se fait questionner, dans la même page, sur sa maison et sur l'algèbre, ou en mathématiques. ${ }^{41}$

Cette croissance spectaculaire s'explique d'abord par des changements majeurs dans le paysage du monde scolaire québécois. La création du tout nouveau ministère de l'Éducation en 1964 en chambarde totalement les structures et engendre, 
temporairement, une période d'incertitude ${ }^{42}$ qui se traduit, momentanément, par une diminution de la production; phénomène inusité, alors que l'offre de nouveaux manuels était en progression constante depuis 1765, moins de manuels nouveaux voient le jour durant la décennie 1960 que durant la précédente. Cette nouvelle donne s'accompagne d'un discours pédagogique tout aussi nouveau : on prétend que le bon professeur n'a pas besoin de manuels, ${ }^{43}$ ou plutôt qu'il doit fabriquer ses propres instruments de travail; le rapport de la commission Parent avait donné le ton : «[...] le maître s'il est compétent et s'il n'est pas surchargé, ne sera pas l'esclave d'un manuel et donnera des cours personnels. " ${ }^{44}$ Mais il y a loin du discours à la pratique : dans les faits, les instituteurs se mettent à produire leurs propres manuels sous forme de cahiers d'exercices, utilisant les nouvelles technologies d'imprimerie maison — " machines à alcool " et " gestetner " ${ }^{45}$ avant de se trouver des éditeurs qui ont vite fait de flairer les avantages économiques de cette nouvelle tendance.

Deux mesures administratives favoriseront l'explosion des cahiers. Le ministère de l'Éducation lance, en 1969, un tout nouveau programme identifié sous la formule " programme-cadre " dont une des caractéristiques est de se contenter de donner des orientations larges - pour ne pas dire vagues — laissant ainsi la porte ouverte à toutes les interprétations de l'enseignant et favorisant l'éclosion de formes variées dans les outils pédagogiques, du manuel traditionnel au cahier qui permet beaucoup de liberté créatrice. ${ }^{46}$ "C'est peut-être l'arrivée des programmes-cadres [...] qui a sonné le glas du livre de classe traditionnel. Beaucoup moins détaillés qu'auparavant et encourageant plus d'initiatives de la part du professeur, les nouveaux programmes entraînèrent l'émergence d'une mode pédagogique favorisant la recherche personnelle de l'élève au détriment de la docilité souvent liée à l'emploi des manuels " constate l'analyste Denis Lebrun. ${ }^{47}$

Serait-ce pour indiquer la marche à suivre que, dès l'année suivante, le ministère de l'Éducation produit lui-même quelques cahiers pour l'enseignement du français? ${ }^{48}$ Cet engouement cède bientôt la place au désenchantement comme le constate un document ministériel en 1977 : "L'intention était manifestement louable et le projet, logique. On voulait que se développe toute la gamme des outils [...]. En pratique, cette intention ne s'est pas concrétisée et s'est avérée trop théorique. " ${ }^{49}$

De cet échec on tirera au moins deux leçons : 1) On redonnera au manuel classique la priorité dans l'ensemble des outils pédagogiques, et on en prend l'engagement formel en 1979; ainsi, dans L'école québécoise - Énoncé de politique et plan d'action, on note : "Le ministère considère nécessaire que les écoles puissent disposer de manuels scolaires de base pour l'enseignement des matières dans toutes les classes du primaire et du secondaire. "; $5^{50}$ 2) On n'abandonnera pas pour autant les cahiers d'exercices qui feront désormais partie du paysage didactique et qui apparaîtront à un tel rythme que le ministère décide qu'à partir du 30 août 1991, ils ne seront plus soumis à l'approbation, ouvrant ainsi la porte toute grande à ce type d'instrument pédagogique. Les chiffres des deux dernières décennies en témoignent éloquemment. Une circulaire administrative du 15 juin 1990 annonce explicitement ce changement d'attitude : "Le matériel didactique de base [entendre le manuel 'classique', le seul soumis à l'approbation] exclura tout document dans lequel l'élève écrit, dessine ou découpe. " ${ }^{51}$ Faut-il y 
voir le constat d'une pratique — l'utilisation massive des cahiers d'exercices — dont le ministère de l'Éducation se dissocierait? Deux ans auparavant, un document officiel émanant du même ministère avait laissé prévoir ce revirement : "Le matériel de base doit être de type permanent et le plus complet possible. Il ne peut se référer en aucune façon de manière explicite à un cahier périssable : renvois, codes, etc. "; et une ligne plus bas on ajoutait cette remarque suave : "Note: Cela n'exclut pas l'existence de tels cahiers. " ${ }^{52}$ Ce changement d'attitude ne signifie en aucun cas une condamnation du cahier d'exercices par les autorités, à preuve ce Guide de sélection des cahiers d'activités ou d'exercices publié par le ministère en 1991 à l'intention des professeurs. ${ }^{53}$

Cette libéralisation permettra d'aborder, en termes crus, des sujets jusque-là tabous, ou tout au moins traités en termes voilés. Ainsi, dans un cahier pour le cours de formation personnelle et sociale publié en 1996, l'élève est appelé à formuler un jugement sur une situation plutôt délicate : «Daniel a 19 ans. Il est incarcéré pour un vol de banque. Sa petite amie vient passer une fin de semaine par mois en prison et ils font l'amour. Le reste du temps, Daniel est l'amant d'un autre détenu âgé de 44 ans qui le protège et le gâte un peu. Il lui arrive également de se masturber avec d'autres détenus quand il prend sa douche. Daniel est-il homosexuel? » Ce texte découle-t-il d'une volonté affichée du ministère de l'Éducation de bannir l'emploi de stéréotypes jusque dans les cahiers d'exercices? ${ }^{54}$ Libéralisation donc, et, avouons-le, avec des effets surprenants, mais les cahiers doivent quand même répondre aux programmes sans quoi les professeurs n'en verront pas l'utilité, ce que reconnaît l'auteur du document en admettant que les élèves auxquels s'adresse ce cahier - 12-13 ans — sont peut-être trop jeunes, mais il ajoute que c'est le seul niveau d'enseignement pour lequel les programmes permettent d'aborder l'homosexualité. ${ }^{55}$

Affaire d'administration gouvernementale, le cahier d'exercices est aussi affaire de commerce. Notant la rapidité avec laquelle les éditeurs ont comblé le vacuum résultant des programmes-cadres de 1969, le ministère de l'Éducation notait en 1979 : " [...] ce vide a abandonné aux maisons d'édition un vaste champ de production mal coordonné de matériel didactique ou de manuels scolaires, lesquels, dans plusieurs milieux, ont vite tenu lieu de programmes. " ${ }^{56}$ Attitude encore plus frappante depuis que ces outils échappent au processus d'approbation et donc ne sont pas fournis gratuitement : les éditeurs en envoient des exemplaires aux professeurs, sachant que ce sont eux, en définitive, qui recommanderont à leurs directions quels titres ont leur préférence et que les parents devront payer. Et le gouvernement n'était pas dupe comme en témoigne un document officiel de 1991 : "Car, même si ce type de matériel didactique est exclu du champ de l'approbation, il est certain que les éditeurs continueront de produire des cahiers d'activités ou d'exercices et de les offrir aux enseignantes et aux enseignants. ${ }^{57} \mathrm{Il}$ n'en demeure pas moins que les éditeurs sentent une pression pour ne pas dire un préjugé favorable des instances gouvernementales envers le manuel classique sans pour autant nier les bienfaits des cahiers d'exercices; le document officiel de 1979 indique que, pour obtenir l'approbation, les manuels devront " proposer des activités diversifiées qui mettent à contribution non seulement les connaissances théoriques de l'élève, mais aussi son application [...] "; ${ }^{58}$ la circulaire de 1991, citée plus haut, se fera encore plus claire : "Les éditeurs ont été informés 
que les manuels scolaires devront présenter à l'avenir des exercices appropriés en quantité suffisante et être de type réutilisable. " Les éditeurs prennent acte : ils vont publier des documents destinés aux professeurs - et donc toujours soumis à l'approbation - mais qui comprennent un certain nombre de "documents reproductibles" qui font office de cahiers d'exercices : sous le texte imprimé on prévoit des espaces où l'enfant écrit ses réponses ou commentaires, documents que le professeur n'a qu'à photocopier pour chacun de ses élèves, pratique d'autant plus officielle qu'on pousse la précision jusqu'à inclure, dans le titre de certains documents, la formule " feuilles à photocopier " ${ }^{59}$; autre avantage : on répond aux parents qui se plaignent du coût élevé des nombreux cahiers qu'il leur faut défrayer. Adaptation officielle aux nouvelles tendances ou retour en arrière? Le même document de 1979, déplorant les abus de photocopie engendrés par les anciens programmes-cadres, dénonçait la pédagogie des "feuilles volantes. " Enfin, faut-il rappeler qu'un des " avantages " économiques de la formule cahiers réside dans l'obligation de les réimprimer chaque année? Il arrive même qu'on utilise la formule " cahier périssable " dans le titre du cahier alors que le livre de l'élève auquel il est associé est formellement identifié comme " cahier non périssable. " ${ }^{61}$

Affaire de normes et affaire de sous, le cahier d'exercices est aussi-espéronsle! - affaire de pédagogie. Notons en premier lieu la diminution, à partir de la décennie 1960, des cahiers d'exercices axés sur la stricte grammaire normative; ${ }^{62}$ si, occasionnellement, on y recourt encore, on demande à l'élève de retranscrire la règle qu'il lui a fallu appliquer dans son exercice. ${ }^{63}$ À l'opposé, on voit se multiplier les cahiers utilisant l'approche ludique. Les mots croisés sont toujours à l'honneur. ${ }^{64}$ Pour les tout-petits on recourt souvent au dessin. ${ }^{65}$ Le cahier peut aussi favoriser les jeux d'équipe comme lorsqu'on découpe les étiquettes d'une page suivant un pointillé pour former des cartons avec un dessin au recto et le mot qui lui est associé au verso. ${ }^{66}$ Au dessin on associe son double, le découpage, comme dans ce cahier de géométrie de 1961 comportant quelques pages imprimées sur carton permettant de découper des figures pour fabriquer des solides. ${ }^{67}$ Enfin, durant certains temps forts comme la préparation à Noël, le cahier se prête non seulement au découpage, mais il permet de faire des montages comme, par exemple, autour du sapin de Noël, réalisations qui peuvent se transformer en décorations pour la classe, voire pour la maison, permettant ainsi aux parents d'apprécier les méthodes utilisées par le professeur;. ${ }^{68}$ autre avantage: les parents voient ainsi à quoi servent les cahiers dont ils défraient les coûts alors, qu'en certains cas, ils se plaignent qu'à la fin de l'année des cahiers n’ont pas été complètement utilisés.

Des méthodes qui ont fait leurs preuves sont encore utilisées, comme demander à l'élève d'appliquer à son milieu immédiat, sa paroisse, des notions générales de géographie. ${ }^{69}$ Larrimage entre des disciplines, jusque-là traitées séparément, continue et prend de l'ampleur, à preuve ces cahiers pour le français qui impliquent des démarches relevant à la fois de la lecture, de la grammaire et de la calligraphie. ${ }^{70}$ Parallèlement, on explore de nouvelles approches. Pour former la main de l'enfant, plutôt que de lui demander de reproduire des modèles de lettres présentées en haut de la page, on lui demande de dessiner par-dessus des lettres déjà tracées en pointillé. ${ }^{11}$ 
Faisant écho au mouvement de l'enseignement des langues étrangères dans la langue concernée, des cahiers pour l'espagnol en tant que langue seconde sont rédigés uniquement dans cette langue. ${ }^{72}$ Enfin, les cahiers, tout comme les manuels, continuent à transmettre des messages pas toujours subliminaux. Le moralisme a toujours sa place — on demande à l'enfant de justifier la punition qu'il subit ${ }^{73}$ — alors qu'ailleurs on préfere s'en tenir à des propos sur l'hygiène. ${ }^{74}$

La grande nouveauté dans les cahiers d'après 1960, par comparaison avec ceux de la période antérieure, demeure le passage des réponses courtes, factuelles, relevant beaucoup plus de la mémorisation que de l'analyse, à des réponses exigeant un développement, obligeant parfois l'élève à s'impliquer personnellement. Ce passage s'est effectué graduellement : en 1979 on se plaint encore que les «travaux dits de recherche se limitent souvent à la simple transposition d'informations trouvées ici et là, sans qu'il y ait eu un effort de compréhension et de synthèse. " ${ }^{75}$ Cette démarche accentue la distanciation entre l'apprenant et l'enseignant, entre le lecteur et le livre : l'élève y gagne en autonomie, le professeur, et le livre, y perdent en autorité.

\section{Conclusion}

Apparaissant tardivement dans le paysage didactique, les cahiers d'exercices ont d'abord été essentiellement des adaptations à des besoins précis en fonction de telle ou telle discipline et n'ont occupé qu'une place marginale dans la panoplie des outils pédagogiques. Le peu d'attention que leur portaient tant les pédagogues que les instances administratives traduit à la fois le peu d'intérêt qu'on leur accordait et l'utilisation minimale qu'on en faisait en classe.

La situation change radicalement à partir de la décennie 1960. À la fois retombée directe et instigateur d'un courant nouveau dévalorisant le manuel classique, ils connaissent un développement spectaculaire et leur succès ne se dément pas même quand, effet de balancier, le discours redore le blason du manuel classique. La position ambigüe du ministère de l'éducation reflète cette évolution : après en avoir fait la promotion, il s'en distancie tout en se gardant bien de le condamner ce qui, de toute façon, ne servirait à rien car le cahier d'exercices fait maintenant partie intégrante de l'enseignement.

L'histoire du manuel scolaire a, jusqu'à tout récemment, intéressé peu de chercheurs, tout au moins au Québec et au Canada, car la France, notamment, fait une heureuse exception avec les travaux du regretté Alain Choppin. Pour ce qui est du champ précis des cahiers d'exercice, une seule étude, celle de Priscilla Boyer précitée. De plus, l'histoire de la pédagogie québécoise — détour incontournable pour comprendre l'histoire du manuel — en est à ses balbutiements. Une fois établi le cadre dans lequel s'est développé ce type d'instrument pédagogique, il reste à en analyser la pertinence pédagogique et son évolution en regard des nouvelles théories pédagogiques.

Enfin, il faudrait s'interroger sur la signification profonde de cette quasi révolution dans le monde du manuel et essayer d'en prévoir le développement futur. Face aux nouvelles technologies utilisées dans l'enseignement — CD-ROM et Internet — quelle 
sera la place du cahier d'exercices et encore plus, quelle est sa signification. Serionsnous en présence d'une tentative — inconsciente? — de redorer le blason de l'imprimé traditionnel et d'amener le jeune à exprimer, par écrit, ses opinions, quitte à devoir ensuite les défendre devant son professeur et ses compagnons?

\section{Notes}

1 Une première version de ce texte a été présentée lors du colloque « School exercise books » à Macerata (Italie) du 26 au 29 septembre 2007.

2 Jean-Joseph Languet, Catéchisme du diocèse de Sens (Québec : Brown \& Gilmore, 1765), $177 \mathrm{p}$.

3 Les données chiffrées de même que les références aux manuels proviennent de la banque Les manuels scolaires québécois que l'on peut consulter à l'adresse url «http.//www.bibl. ulaval.ca/ress/manscol». Conçu comme un centre de documentation virtuel destiné à ceux que l'histoire du manuel scolaire québécois intéresse, ce site offre plusieurs pages informatives facilitant la compréhension de cet outil pédagogique : sources imprimées, sources manuscrites, historiographie, bibliographie, textes de lois, etc. Sa principale section demeure toutefois le catalogue des manuels publiés au Québec, soit 23000 documents qui comprennent à la fois les éditions princeps et les réimpressions tant des manuels destinés directement aux élèves que ceux destinés aux maitre; le moteur de recherche permet de les regrouper par discipline, auteur, date, éditeur de même qu'on peut isoler les manuels destinés aux anglophones, aux Amérindiens sans oublier les éditions princeps, les manuels approuvés et ... les cahiers. Dans ce texte, je ne prends en compte que les seules éditions princeps destinées aux élèves sans les « livres du maître ». Les données dont on y fait état dans ce texte proviennent d'une interrogation de la banque alors qu'elle ne contenait que 22000 documents.

4 Pour une synthèse de l'histoire du manuel scolaire au Québec on consultera Paul Aubin, "Le manuel scolaire québécois entre l'ici et l'ailleurs ", Monique Lebrun, Le manuel scolaire d'ici et d'ailleurs, d'hier à demain (Québec : Presses de l'Université du Québec, 2007), 25-62.

5 Le catalogue à la base de cette recherche recense les manuels dont une des phases de production a été réalisée au Québec; or, depuis une cinquantaine d'années, il ne s'y est pratiquement pas publié de manuels pour anglophones, leurs écoles utilisant presque uniquement des manuels produits à l'extérieur, ce qui explique la faible représentation du secteur anglophone dans ce tableau.

6 J. L. Figler, Figler's jewish writing method - No. 1-6 (Montréal : Figler, c1917), 24 p. (Bilingue : Anglais et Hébreu).

7 Christiane Pageau et Paulusi Uqittuq, Ecris - Lis - Mon premier livre d'esquimau, 2 éd., (S.1., Ministère des richesses naturelles, 1967), 44-45.

8 La subite augmentation de la décennie 1890-13\%— est artificielle : on publie des séries de cahiers pour la calligraphie, chaque série offrant un cahier différent pour chaque niveau du cours.

9 G. Rhyn, Mon histoire sainte : album à colorier, (Montréal : Librairie pédagogique, n.d.), 11.

10 Edmond-Marie Templé, Méthode nationale de dessin : premier cours : feuilles exercices (Montréal : C.O. Beauchemin, n.d.), 1 portefeuille.

11 Frères de l'instruction chrétienne, Mon devoir d'histoire du Canada : Découvreurs et pionniers : $4^{e}$ et $5^{e}$ années (La Mennais (Laprairie) : Frères de l'instruction chrétienne, n.d.), 20. L'Amérique (moins le Canada) - cahier de travaux pratiques $n^{\circ} 3$ (Montréal : Centre de psychologie et de pédagogie, c1957), 5. «Dans un travail sur Montcalm, 
on fournit, en vrac, les réponses, l'élève n'ayant qu'à les placer au bon endroit pour compléter les réponses déjà partiellement énoncées.

12 Nive Voisine, Les Frères des Écoles chrétiennes au Canada-Tome 1-La conquête de l'Amérique 1837-1888 (Québec: Anne Sigier, 1987), 351.

13 "Un vice dans nos Écoles ", Journal de l'instruction publique 13, 7 (juil. 1869) : 89.

14 Frère Marie-Victorin, "Les cercles des jeunes naturalistes ", Le Devoir, 22, 110 (13 mai 1931) : 2 .

15 Frères des écoles chrétiennes, Cours de calligraphie commerciale : $3^{e}$ ordre (Montréal : s.n., c1908), 11.

16 Frères des écoles chrétiennes, Etude de la comptabilité : $10^{\circ}$ année: cahier $n^{\circ} 3 b$-The study of accounting: 10th year: exercise book no $3 b-3 a \& 3 b$ (Québec : Centre pédagogique, n.d.), $117 \mathrm{p}$.

17 Frère Meldas-Cyrille, Mon cahier de religion : neuvième année (Montréal : Frères des écoles chrétiennes, c1944), 52.

18 Frères de l'instruction chrétienne, Mon devoir d'histoire du Canada: Mon pays : Régime français : $8^{e}$ et $9^{e}$ années : $1^{r e}$ partie (La Mennais (Laprairie) : Frères de l'instruction chrétienne, n.d.), 80.

19 Frères des écoles chrétiennes, Cahier de calcul : addition (S.l., s.n., c1882), 11.

20 W.J. McBriarty, The french-canadian's guide exercises in english grammar [...] Fourth series [...] 50 exercises (Québec : Montréal, Pedagogia, [1936]), n.p.

21 Frères des écoles chrétiennes, 50 devoirs sur la syntaxe (Montréal : Frères des écoles chrétiennes, 1937, c1925), 50.

22 Gertie Kathleen Hart, Méthode directe de conversation anglaise - cahier d'exercices de conversation écrite - 1a (Québec : Presses de l'Université Laval, 1954), 12.

23 Soeurs de l'Assomption de la Sainte Vierge, Mon cahier d'enfant du bon Dieu - 6 année (Nicolet : s.n., 1939), n.p.; Soeurs de l'Assomption de la Sainte Vierge, Mon dimanche avec la sainte Eglise (S.l., s.n., n.d.), 25; Soeurs de l'Assomption de la Sainte Vierge, Mes cahiers d'enfant du bon Dieu - 2ème année (Nicolet : Soeurs de l'Assomption de la S.V., 1939), 12.

24 Frères des écoles chrétiennes, Cours de géographie à l'usage des écoles chrétiennes : exercices cartographiques ou cartes muettes à compléter et à reproduire: cahier $n^{\circ} 1$ en rapport avec le cours élémentaire (S.l., s.n., c1882), 1; Frères des écoles chrétiennes, Cours de géographie à l'usage des écoles chrétiennes : exercices cartographiques ou cartes muettes à compléter et à reproduire : cahier $n^{\circ} 3$ en rapport avec le cours supérieur (S. 1., s.n., c1882), 7.

25 Joseph-Pierre Brutto, Cahier de cartographie : classes de 4me et 5 me (Montréal : Librairie pédagogique, n.d.), $20 \mathrm{p}$.

26 Géographie générale (physique et humaine) : cahier de travaux pratiques no 1 (Montréal: Centre de psychologie et de pédagogie, c1957), 3.

27 Mon cahier de cartes muettes - 4e année (Montréal : Centre de psychologie et de pédagogie, c1954), 54.

28 Frères de l'instruction chrétienne, Mon devoir d'histoire du Canada: Mon pays : $7^{e}$ année : Nouveau programme (Laprairie : Frères de l'instruction chrétienne, n.d.), 41. L'Amérique (Le Canada) : $11^{e}$ année - cahier de travaux pratiques n $n^{\circ}$ 4, (Montréal : Centre de psychologie et de pédagogie, [1959], c1957), 36.

29 Marcel Sicotte, Cahier de chimie, $11^{e}$ et $12^{e}$ années (Montréal : Centre de psychologie et de pédagogie, c1957), 39.

30 Gérard Beaudry, 1-2-3, cahier de calcul : première année - première partie (Montréal : Beauchemin, 1959), $40 \mathrm{p}$.

31 Frères du Sacré-Coeur, Mon cahier de calcul : 1 ire année - No 2 (Montréal : Procure des frères du Sacré-Cour, c1947), 23.

32 Soeurs de l'Assomption de la Sainte Vierge, Au pays des contes - cahier d'exercices employé avec Le trésor des jeunes conteurs (S. 1., W.J. Gage, n.d.), 34. 
33 Frères des écoles chrétiennes, Découvreurs et pionniers : histoire du Canada, cahier d'exercices sur le manuel de $4^{e}$ et $5^{e}$ années (Montréal : Frères des écoles chrétiennes, La librairie des écoles, c1961), 31.

34 W. J. McBriarty, Key to guide exercises in english grammar (Based on "The frenchcanadian's guide in english grammar") - Fourth series (Québec : s.n., c1946), 81 p.

35 L. Pelletier, Méthode facile pour apprendre rapidement l'anglais - Livre du maître du cahier $H-7^{e}$ et $8^{e}$ années (S.1., s.n., c1945), 39 p.

36 Soeurs de l'Assomption de la Sainte Vierge, Mes cahiers d'enfant du bon Dieu - $3^{e}$ et $4^{e}$ années - Guide du maître (Nicolet : Sœurs de l'Assomption de la S.V., 1952), 20 p.

37 Frères de l'instruction chrétienne, Mon devoir d'histoire du Canada: Découvreurs et pionniers: $4^{e}$ et $5^{e}$ années (La Mennais (Laprairie) : Frères de l'instruction chrétienne, n.d.), 20.

38 Frères des écoles chrétiennes, 50 devoirs sur les verbes : $4^{e}, 5^{e}$ et $6^{e}$ années : conjugaisons, orthographe, syntaxe (Montréal : s.n., 949, rue Côté, 1946, c1925), 50 p. Comité catholique du Conseil de l'instruction publique, 5 mai 1953, p. 169.

39 Julien Biron et al., Français 2 - Baluchon à l'atelier - Cahier d'activités - $2^{e}$ partie (Outremont : Lidec, c1985), 28. Julien Bergeron et al., Français 2 - Baluchon à l'atelier Cahier d'activités - $2^{e}$ partie - Immersion (Outremont : Lidec, c1987), 28.

40 Aline Charlebois, Ouvre un livre pour voir! - Education des adultes - Alphabétisation Cahier B (S.l., Marie-France, c1993), 21, 23.

41 Simone Bussières, Mon cahier de vacances - $3^{e}$ année (Montréal : Centre de psychologie et de pédagogie, circa 1965), 63 p. Ken Dolphin, et Jean-Paul Gagnon, Ami-mots en vacances - Cahier de lecture d'été des amis du club ami-mots - Niveau 2 (Saint-Laurent : Trécarré, c1990), 44. Alma Albert, Ami-math en vacances - Cahier d'activités mathématiques d'été - Niveau 1 (Saint-Laurent : Trécarré, c1991), 62 p.

42 Pour une analyse de ces changements, voir Rapport de la commission royale d'enquête sur l'enseignement dans la province de Québec - Première partie ou Tome I-Les structures supérieures du système scolaire (S.l., s.n., 1965), xiii, 121 p.

43 Le débat sur la place du manuel dans l'enseignement n'est pas nouveau. Un périodique destiné aux professeurs anglo-québécois s'y intéresse au $19^{\mathrm{e}}$ siècle ("The Teacher is the Book ", The Journal of education 13, 6 (June 1869) : 97-98) et on cherche encore un consensus sur ce sujet (Astrid Berrier, "Enseigner avec un manuel ou sans? ", Québec français 113 (printemps 2000) : 38-39).

44 Rapport de la commission royale d'enquête sur l'enseignement dans la province de Québec -Deuxième partie - Les structures pédagogiques du système scolaire-A-Les structures et les niveaux de l'enseignement (S.l., s.n., [1964]), 110.

45 Un document officiel le constate : «Dans la plupart des cas, pour donner leurs cours, les enseignants se sont mis en quête d'une documentation puisée aux sources les plus diverses, en y ajoutant des notes personnelles qu'ils rédigeaient et reproduisaient à l'intention de leurs élèves. " (L'école québécoise-Énoncé de politique et plan d'action (S. l., Ministère de l'Éducation, 1979), 104).

46 L'approbation des manuels scolaires (S.1., Ministère de l'Éducation, 1993), 1.

47 Denis Lebrun, "L'édition du livre scolaire au Québec : un tournant ", Éducation Québec 10, 2 (oct. 1979) : 11.

48 Français 112 - Secondaire I (Québec: Ministère de l'Éducation, c1970), 5 fascicules.

49 L'enseignement primaire et secondaire au Québec - Livre vert (Québec : Ministère de l'Éducation, 1977), 21. Pour l'instant, les cahiers d'exercices sont officiellement entérinés par le ministère car on les range parmi « les instruments pédagogiques » (p. 101).

50 L'école québécoise - Énoncé de politique et plan d'action (S.l., Ministère de l'Éducation, 1979), 107.

51 Circulaire administrative AG-054-89-009. 
52 Guide de conception et d'évaluation du matériel didactique destiné aux élèves en difficulté d'apprentissage (Primaire et secondaire) (S.l., Ministère de l'Éducation, 1989), 10.

53 Guide de sélection des cahiers d'activités ou d'exercices (Québec : Ministère de l'Éducation, 1991), 34.

54 Guide pour adultes avertis - Document servant à reconnaître les stéréotypes discriminatoires dans les cahiers d'exercices et le matériel didactique complémentaire (S.I., Ministère de l'Éducation, 1991), 22 p.

55 Martial Boucher, Au printemps de la vie - Formation personnelle et sociale - $1^{\text {er }}$ cycle $d u$ secondaire - Cahier d'activités et de contenu (Montréal : Lidec, c1996), 97. Marie Allard, « Daniel, prisonnier bisexuel et vedette d'un manuel scolaire-Le contenu d'un cahier d'exercice de secondaire est dénoncé ", Le Soleil, 30 août 2003, A7. Même si, comme le rappelle Priscilla Boyer, les « auteurs [des cahiers] n'ont donc pas d'obligation légale de respecter le programme officiel ", il n'en demeure pas moins qu'ils ne peuvent s'en éloigner à leur guise. (Priscilla Boyer, «Le cahier d'activités grammaticales-Témoin privilégié de l'évolution de la didactique de la grammaire ", Monique Lebrun, Le manuel scolaire d'ici et d'ailleurs, d'hier à demain (Québec : Presses de l'Université du Québec, 2007), 2.

56 L'école québécoise - Énoncé de politique et plan d'action (S.1., Ministère de l'Éducation, 1979), 88.

57 Guide de sélection des cahiers d'activités ou d'exercices - Primaire - Secondaire — Document d'information (S.1., Ministère de l'Éducation, 1991), 1.

58 Danielle Dion-McKinnon et Pierre Lalongé, Animer notre histoire - Guide méthodologique et documents reproductibles (Montréal : ERPI, c1985), xii, 242 p.

59 Pierrette Gaudreau, Collection alouette - 6 année - Matériel complémentaire - Feuilles à photocopier (Montréal : Toronto, Guérin, c1987), 8 p.

60 L'école québécoise - Énoncé de politique et plan d'action (S.l., Ministère de l'Éducation, 1979), 106-107.

61 Suzanne Roy et Yolande Tremblay, Collection Et si on lisait ... - Troisième année - Cloé 2 - Cahier périssable (Boucherville : Éditions françaises, c1985), 48 p. Suzanne Roy et Yolande Tremblay, Collection Et si on lisait ... - Troisième année - Cloé 1 - Cabier non périssable (Boucherville : Éditions françaises, c1985), 48 p.

62 Alain de Bray, Exercices de grammaire française - cahier $C$ (Montréal : Brault et Bouthillier, c1970, c1966), $147 \mathrm{p}$.

63 René Bureau et André Clas, Méthode active d’orthographe - Cahier A (S.1., Beauchemin, c1969), 22.

64 Diane Beaudoin, L'espace - Préscolaire - 5 ans - Dossier reproductible (Montréal-Nord : Marie-France, c2003), 21, 34 p.

65 Lise Bernard, Habilités et connaissances en lecture - cahier d'activités (Boucherville : Graficor, c1988), 2.

66 Suzanne Guillemette et Nicole Raymond, Mon cahier d'étiquettes-mots (Boucherville : Graficor, c1993), [28] p.

67 Gérard Tessier et Raoul Beaugrand, Initiation à la géométrie : géométrie intuitive : cahier de recherches (Montréal : Centre de psychologie et de pédagogie, c1961), $54 \mathrm{p}$.

68 Pierrette Gaudreau, Collection alouette - $3^{e}$ année - Matériel complémentaire - Feuilles à photocopier (Montréal/Toronto : Guérin, c1987), 13 p. Pierrette Gaudreau, Collection alouette - 3 - Guide de l'enseignante (Montréal/Toronto : Guérin, c1985), 43.

69 Jean-Bernard Racine et André Poulin, Mon milieu et ma région - Géographie - 4 année Cahier d'exercices (Laval : Éditions FM, c1968), 31.

70 Raymond Berthiaume, Raymond et Raymond Paradis, Les récits du dimanche Exercices de lecture, de compréhension de textes et de grammaire - $2^{c}$ année du primaire (Montréal-Nord : Marie-France, c1994), 49. 
71 J'apprends les minuscules (Montréal-Nord : Marie-France, c1984), 9.

72 Denise Lugo-Perron, Cuaderno de ejercicios escritos (S.l., Guérin, c1996), 53.

73 Raymond Berthiaume, J'explore en lisant - 2e cycle-Cahier A (S.1., Marie-France, c2004), 56.

74 Germaine Pouliot, Monique Pouliot, et Suzelle Roberge, Cataradi 3 - Cahier d'activités $2^{2}$ semestre (Anjou : CEC, c1990), 30.

75 L'école québécoise - Énoncé de politique et plan d'action (S.1., Ministère de l'Éducation, 1979), 105. 